\title{
Effects of safflower seed extract supplementation on oxidation and cardiovascular risk markers in healthy human volunteers
}

\author{
Naoto Koyama $^{1}$, Katsuya Suzuki ${ }^{1}$, Yasushi Furukawa ${ }^{2}$, Harumi Arisaka ${ }^{1}$, Tetsuya Seki ${ }^{1}$, \\ Kanna Kuribayashi ${ }^{1}$, Koichi Ishii ${ }^{1}$, Eiji Sukegawa ${ }^{3}$ and Michio Takahashi ${ }^{1}$ \\ ${ }^{1}$ Research Institute for Health Fundamentals, Ajinomoto Co., Inc., 1-1, Suzuki-cho, Kawasaki-ku, Kawasaki 210-8681, Japan \\ ${ }^{2}$ Human Resources Department, Wellness Promotion Center, Ajinomoto Co., Inc., 1-1, Suzuki-cho, Kawasaki-Ku, Kawasaki \\ 210-8681, Japan \\ ${ }^{3}$ Health Services Development Department, Ajinomoto Co., Inc., 1-15-1, Kyobashi, Chuo-Ku, Tokyo 104-8315, Japan \\ (Received 11 January 2008 - Revised 31 March 2008 - Accepted 23 May 2008 - First published online 1 July 2008)
}

\begin{abstract}
We previously demonstrated that safflower seed extract (SSE) and its major antioxidant constituents, serotonin hydroxycinnamic acid amides, suppressed LDL oxidation in vitro, decreased plasma autoantibody titres to oxidized LDL and attenuated atherosclerotic lesion formation in apoE-deficient mice. In this report, we examined whether SSE, rich in serotonin derivatives, could affect markers of oxidative stress, inflammation and aortic stiffness in healthy human subjects. Twenty Japanese male volunteers were studied at baseline, after $2.1 \mathrm{~g}$ SSE supplementation daily (providing $290 \mathrm{mg}$ serotonin derivatives/d) for 4 weeks, and after a 4-week washout period. Significant reductions in circulating oxidized LDL, autoantibody titres to malondialdehyde-modified LDL, the soluble form of vascular cell adhesion molecule-1 (sVCAM-1), and urinary 8-isoprostane were observed after a 4-week intervention. Although there were no statistically significant differences in blood pressure or brachial-ankle pulse wave velocity (baPWV), an index of arterial stiffness, baPWV was lower than baseline in eleven of twenty subjects and was accompanied by a reduction in blood pressure. Statistically significant negative correlations were observed between the extent of initial cardiovascular risk markers (autoantibody titres, 8-isoprostane, sVCAM-1 and baPWV) and the effect of intervention. This suggested that individuals with elevated oxidative stress, inflammation, and/or arterial stiffness may receive more benefit from SSE supplementation.
\end{abstract}

Safflower seed extract: Serotonin derivatives: Human studies: 8-Isoprostane: Oxidized LDL

Many epidemiological studies have shown inverse relationships between consumption of diets rich in polyphenols and cardiovascular risk. Because much evidence indicates that an increased oxidative insult, such as oxidative modification of LDL, is deeply involved in the development of atherogenesis, it seems reasonable to attribute the cardioprotective effect of dietary polyphenols to their potent antioxidant activity. This assumption indeed appears to be supported by a number of in vitro and animal studies, but has not been substantiated to date by results from human studies ${ }^{(1-3)}$.

Most efforts to demonstrate health benefits of dietary polyphenols have been focused on flavonoids, because flavonoids are one of the most abundant polyphenols in our foods and beverages. Besides flavonoids, there are many other dietary compounds that may exert significant antioxidant effects both in vitro and in vivo. Indoles such as serotonin, melatonin, tryptophan, indole-3-carbinol, pyridoindoles, etc., occur endogenously and/or in many plants, and may comprise a family of radical scavengers ${ }^{(4)}$. It has been suggested that some pharmacological effects of particular indoles result from their antioxidant properties ${ }^{(5,6)}$. Serotonin hydroxycinnamic acid amides (e.g. $N$-( $p$-coumaroyl)serotonin (CS); $N$-feruloylserotonin) are potent scavengers against free radicals ${ }^{(7,8)}$. We previously demonstrated that serotonin derivatives and safflower seed extract (SSE) that contain them as major antioxidants protected LDL from copper- or azo-compound-induced in vitro oxidation, and attenuated atherosclerotic lesion formation in apoE-deficient mice $^{(9)}$. A concomitant decrease in autoantibody titres to oxidized LDL (oxLDL) and lipid peroxides in mouse plasma suggested their in vivo antioxidant effects as a possible mechanism for their anti-atherogenicity.

While the serotonin derivatives are detected in several plants, to the best of our knowledge, safflower (Carthamus tinctorius L.) seed is one of the richest sources of these indolic polyphenols ${ }^{(8,9)}$. While safflower seeds are used in herbal medicines in some countries, they are produced worldwide mainly for edible oil production. As the defatted seeds, which are abundant in the serotonin derivatives, are generally not utilized for human use, their beneficial effect on human health has therefore not been scientifically clarified.

\footnotetext{
Abbreviations: anti-MDA-LDL, autoantibody titres to malondialdehyde-modified LDL; baPWV, brachial-ankle pulse wave velocity; BP, blood pressure; CS, $N$-( $p$-coumaroyl)serotonin; KHC rabbit, Kurosawa and Kusanagi Hypercholesterolemic rabbit; MCP-1, monocyte chemotactic protein-1; oxLDL, oxidized LDL; PWV, pulse wave velocity; sICAM-1, soluble form of intercellular adhesion molecule-1; SSE, safflower seed extract; sVCAM-1, soluble form of vascular cell adhesion molecule-1.

* Corresponding author: Naoto Koyama, fax +81 44211 7609, email naoto_koyama@ajinomoto.com
} 
The objective of the present study was to evaluate the potential of SSE, which is rich in serotonin derivatives, to act as an antioxidant in man and to estimate which populations are more likely to receive benefit from SSE, if any, for future study. For this purpose, we conducted a pilot human trial in which we compared pre- and post-intervention values of various oxidation biomarkers including the following: 8-isoprostane, which is currently considered a reliable marker of systemic oxidative stress ${ }^{(10)}$; circulating oxLDL and its autoantibody titres, which are evidence that LDL oxidation takes place in vivo and have been demonstrated to be higher in patients with coronary artery disease ${ }^{(11,12)}$; and inflammation indices, because oxidative stress is known to be deeply involved in this process. In addition to these biochemical parameters, we examined the effect of SSE on pulse wave velocity (PWV), a non-invasive measure of arterial stiffness. Recent studies have demonstrated that increased arterial stiffness is an independent risk factor of $\mathrm{CVD}^{(13,14)}$. Improvements in arterial stiffness were found by some dietary interventions; such as in our recent results that showed attenuation of aortic PWV in cholesterol-fed Kurosawa and Kusanagi Hypercholesterolemic (KHC) rabbits fed on a diet supplemented with SSE or serotonin derivatives for 4-8 weeks $^{(15)}$. This prompted us to look into the effect of SSE on this parameter.

\section{Subjects and methods}

\section{Study protocol and subjects}

Healthy Japanese male volunteers ( $n$ 20) from Ajinomoto Co. Inc. (Kawasaki, Japan) were recruited and were studied at baseline, after supplementation with SSE for 4 weeks and at the end of a 4-week washout period (for personal reasons, one subject could not participate in the last measurement). The study protocol was approved by the institutional review board and written informed consent was obtained from all subjects. Subjects were recruited if they fulfilled the following criteria: age 30 years or older; no history of CVD; nondiabetic; with or without mild lipid disorder (total cholesterol $\leq 6.2 \mathrm{mmol} / \mathrm{l}$ or LDL-cholesterol $\leq 4.1 \mathrm{mmol} / \mathrm{l}$ ); with or without mild hypertension (systolic blood pressure (BP) $<160 \mathrm{mmHg}$ or diastolic BP $<100 \mathrm{mmHg}$ ); not taking antihyperlipidaemic, antihypertensive or anti-inflammatory medications; no liver problems; no history of drug allergy. Subjects had a mean age of 37.3 (SD 6.8) years and a BMI of $23.7(\mathrm{SD} 3.7) \mathrm{kg} / \mathrm{m}^{2}$. All subjects received SSE in capsules for 4 weeks (twice a day, five capsules each time, within $30 \mathrm{~min}$ of breakfast and supper). Total daily SSE intake was $2 \cdot 1 \mathrm{~g}(290 \mathrm{mg} / \mathrm{d}$ as serotonin derivatives $)$, and the participants subsequently underwent a 4-week washout phase. Subjects were asked to record the consumption of the SSE supplements and their health status every day throughout the study. In addition, they were advised to maintain their lifestyles, but not to consume other antioxidants, antilipidaemics or antihypertensive dietary supplements such as vitamin E or C, soya isoflavone concentrates, green tea extracts, pycnogenol and related formulations, fish or flaxseed oil supplements, or angiotensin-converting-enzyme inhibitory peptide concentrates.

\section{Safflower seed extract preparation}

Defatted safflower seeds were provided by Oilseeds International Ltd (San Francisco, CA, USA). A total of $100 \mathrm{~kg}$ of the defatted safflower seeds were first washed with water (2000 litres) at $30^{\circ} \mathrm{C}$ for $30 \mathrm{~min}$ with stirring, followed by centrifugation, and subsequently the residue was extracted with $60 \%(\mathrm{v} / \mathrm{v})$ ethanol (1500 litres) at $60^{\circ} \mathrm{C}$ for $60 \mathrm{~min}$ with stirring. This procedure was repeated twice more and the extracts obtained were combined, filtered and then concentrated in vacuo at $50-60^{\circ} \mathrm{C}$ after the addition of an equivalent amount of $\gamma$-cyclodextrin (CAVAMAX W8 Food; CycloChem Co. Ltd, Kobe, Japan) as dry solids in the filtrate to facilitate concentration. As a result, this SSE preparation contained $50 \%(\mathrm{w} / \mathrm{w})$ of $\gamma$-cyclodextrin. The concentrated defatted SSE was heat-sterilized at $88^{\circ} \mathrm{C}$ for $60 \mathrm{~min}$, airdried at $60^{\circ} \mathrm{C}$ for $15 \mathrm{~h}$, then pulverized. A total of $6 \mathrm{~kg}$ of powdered SSE was finally obtained. The total polyphenol content of the SSE, determined by the Folin-Ciocalteau method ${ }^{(16)}$, was $143 \mathrm{mg}$ (as CS equivalent)/g SSE. Contents of the major safflower seed phenolics in the extract were also determined by the HPLC method described in our earlier paper ${ }^{(9)}$ and are shown in Table 1 together with the macronutrient composition of the SSE. The SSE powder was capsulated in hard gelatin capsules $(210 \mathrm{mg} / \mathrm{capsule})$ and served as an experimental dietary supplement.

\section{Sample collection and haemodynamic measurements}

Haemodynamic measurements and blood/urine samples were collected in the morning at baseline and every 4 weeks thereafter (0, 4, 8 weeks). Subjects were asked to fast overnight and refrain from smoking until the measurements and sample collections were done. After a urine sample (second morning urination) was collected upon the subject's arrival for testing, body weight, and brachial BP/brachial-ankle pulse wave velocity (baPWV) were measured in that order in a temperature-controlled room maintained at $25^{\circ} \mathrm{C}$. A blood sample was then taken. All haemodynamic measurements were conducted with subjects in the supine position after their heart rate had settled to a resting level. BP and baPWV were measured simultaneously with an automated device, form PWV/ABI (BP-203RPE II; Omron Colin,

Table 1. Composition of safflower seed extract (SSE)

\begin{tabular}{lr}
\hline & \multicolumn{1}{c}{ SSE } \\
\hline Energy $(\mathrm{kJ} / 100 \mathrm{~g})$ & $1642 \cdot 5$ \\
Protein $(\mathrm{g} / 100 \mathrm{~g})$ & $9 \cdot 8$ \\
Fat $(\mathrm{g} / 100 \mathrm{~g})$ & $3 \cdot 4$ \\
Carbohydrate $(\mathrm{g} / 100 \mathrm{~g})$ & 80.2 \\
Ash $(\mathrm{g} / 100 \mathrm{~g})$ & $3 \cdot 7$ \\
Dietary fibre $(\mathrm{g} / 100 \mathrm{~g})$ & $0 \cdot 8$ \\
Serotonin derivatives $(\mathrm{mg} / \mathrm{g})$ & \\
CS & 32.2 \\
FS & 31.7 \\
CS-glc & 48.5 \\
FS-glc & 25.7 \\
Total & 138.1 \\
\hline
\end{tabular}

CS, $N$-( $p$-coumaroyl)serotonin; CS-glc, CS monoglucoside; FS, $N$-feruloylserotonin FS-glc, FS monoglucoside. 
Tokyo, Japan). The detailed methodology for measurement of baPWV has been described elsewhere ${ }^{(17)}$. In brief, four pneumatic pressure cuffs, two electrocardiogram electrodes and a microphone for detecting heart sounds were attached at both arms and ankles, on both wrists, and on the left edge of the sternum, respectively, to record the volume waveform for the brachial and ankle arteries. The baPWV was automatically calculated as the length of an arterial segment between brachium and ankle, which was automatically calculated from the body height, divided by the transit time of the pulse wave. Left side measurements of baPWV and BP were taken for further analysis.

\section{Blood and urinary biochemical parameters}

Plasma glucose, serum lipids (total cholesterol, LDL-cholesterol, HDL-cholesterol, TAG), serum or urinary parameters for liver and renal function (total protein, aspartate aminotransferase, alanine aminotransferase, lactate dehydrogenase, $\gamma$-glutamyltranspeptidase, albumin, blood urea nitrogen, urinary creatinine), blood monoamines (plasma adrenaline, noradrenaline, dopamine, whole-blood serotonin) and 5-hydroxyindoleacetic acid (a major metabolite of serotonin) were measured by SRL Inc. (Tokyo, Japan) using their standard laboratory protocols.

\section{Oxidative stress status}

Serum autoantibody titres to malondialdehyde-modified LDL (anti-MDA-LDL) were measured by ELISA. Protein carbonyls were measured in serum according to the method of Levine et al. ${ }^{(18)}$. Urinary 8-isoprostane and 8-hydroxydeoxyguanosine were measured using an 8-Isoprostane EIA Kit (Cayman Chemical, Ann Arbor, MI) and a New 8-OHdG Check ELISA (Nikken SEIL Co. Ltd, Fukuroi, Japan), respectively. Antioxidant enzyme activities, superoxide dismutase and paraoxonase, were measured by the improved nitrite $\operatorname{method}^{(19)}$ and colorimetric assay, respectively. All these parameters were measured by SRL Inc. OxLDL was measured in serum as 4E6 monoclonal antibody-reactive substances using an Oxidized LDL Competitive ELISA Kit (Mercodia, Uppsala, Sweden). Thiobarbituric acid-reactive substances were measured in serum and LDL according to the method of Ohkawa et al. ${ }^{(20)}$. LDL was isolated from the collected blood sample in accordance with the procedures of Chung et al. ${ }^{(21)}$.

\section{Proinflammatory markers}

The serum soluble form of vascular cell adhesion molecule-1 (sVCAM-1), the soluble form of intercellular adhesion molecule-1 (sICAM-1) and monocyte chemotactic protein-1 (MCP-1) were measured by SRL Inc. using Quantikine Immunoassay kits (R\&D Systems, Minneapolis, MN, USA). High-sensitivity C-reactive proteins were also measured at SRL Inc. by nephelometry.

\section{Statistical analysis}

Results were given as means and their standard deviations unless otherwise noted. All data analyses were conducted with the use of SigmaStat 3.11 (Systat Software Inc., San Jose, CA, USA). Differences between values at each timepoint were evaluated using one-way repeated ANOVA with post hoc comparison by the Tukey test. Pre- and postsupplementation variables were compared with the paired $t$ test. Correlations between initial risk values and the effects of SSE supplementation on corresponding risk factors were analysed by Pearson's correlation analysis. $P<0.05$ was considered statistically significant.

\section{Results}

Effects on measures of safety, serotonin and its metabolite

All twenty subjects completed the trial without any significant subjective symptoms or change in body weight (Table 2). There were no significant changes from baseline (week 0) to the completion of SSE supplementation (week 4) in blood biochemical parameters for hepatic and renal function (data not shown). There were also no significant differences between baseline and week 4 in blood lipids and glucose (Table 2). Whole-blood serotonin was significantly elevated from baseline at week 4 and subsequently declined during the washout period though it remained significantly higher than the baseline level at week 8 (Table 2). This elevation at week 4 and the subsequent decline at week 8 were observed in most subjects (nineteen of the twenty, and sixteen of eighteen subjects, respectively). Although not statistically significant, the plasma level of a serotonin metabolite, 5-hydroxyindoleacetic acid, also showed a similar pattern $(P=0.054$ at week $4 v$. baseline). The change was characteristic for serotonin and 5-hydroxyindoleacetic acid. No such elevation was observed in other biogenic monoamines (adrenaline, noradrenaline and dopamine).

\section{Effects on brachial-ankle pulse wave velocity and blood pressure}

There were no statistically significant changes in baPWV and BP between pre- and post-supplementation when analysis was conducted on the entire cohort (Table 3).

\section{Effects on oxidative stress markers}

Significant $(P<0.05)$ reductions from baseline (week 0$)$ at the end of SSE supplementation (week 4) were observed for the following oxidative parameters (Table 4): serum oxLDL, serum anti-MDA-LDL and urinary 8-isoprostane. Mean week 8 values of these parameters were still lower than at baseline; however, there was a $\mathrm{V}$-shaped pattern where the mean value at week 4 was the lowest. Week 4 values of oxLDL, anti-MDA-LDL and 8-isoprostane were smaller than at baseline in fifteen, sixteen and twenty of the twenty subjects, respectively. No significant changes were seen in the other parameters. A slight, but significant, increase of serum paraoxonase activity was seen only at week 8 .

\section{Effects on inflammation markers}

Serum sVCAM-1 significantly decreased at week $4(P<0.05$ $v$. baseline) and returned to baseline at the end of the 
Table 2. Effects of safflower seed extract supplementation on body weight, blood lipids, blood glucose, serotonin and its metabolite, and other biogenic monoaminesł

(Mean values with their standard errors)

\begin{tabular}{|c|c|c|c|c|c|c|}
\hline & \multicolumn{2}{|c|}{ Week $0(n 20)$} & \multicolumn{2}{|c|}{ Week 4 ( $n$ 20) } & \multicolumn{2}{|c|}{ Week 8 (after washout, $n$ 19) } \\
\hline & Mean & SEM & Mean & SEM & Mean & SEM \\
\hline \multicolumn{3}{|l|}{ Cholesterol $(\mathrm{mmol} / \mathrm{l})$} & $71 \cdot 2$ & $2 \cdot 8$ & $71 \cdot 3$ & $3 \cdot 1$ \\
\hline $\begin{array}{l}\text { Cholesterol }(\mathrm{mmol} / \mathrm{l}) \\
\text { Total }\end{array}$ & 5.0 & 0.2 & 4.9 & 0.2 & $5 \cdot 3$ & 0.2 \\
\hline LDL & 2.9 & 0.1 & 2.9 & 0.2 & $3 \cdot 3$ & 0.2 \\
\hline $\mathrm{HDL}$ & 1.6 & 0.1 & 1.5 & 0.1 & 1.6 & 0.1 \\
\hline TAG (mmol/l) & 1.2 & 0.1 & 1.2 & 0.2 & 1.3 & 0.2 \\
\hline FBG $(\mathrm{mmol} / \mathrm{l})$ & 5.4 & 0.1 & $5 \cdot 3$ & 0.1 & $5 \cdot 3$ & 0.1 \\
\hline Serotonin $(\mathrm{ng} / \mathrm{ml})$ & 93.6 & $6 \cdot 1$ & $114 \cdot 6^{*}$ & $6 \cdot 1$ & $100 \cdot 4^{*} \dagger$ & $7 \cdot 0$ \\
\hline 5-HIAA (ng/ml) & 3.6 & 0.2 & 4.6 & 0.6 & 3.7 & 0.2 \\
\hline Adrenaline $(\mathrm{pg} / \mathrm{ml})$ & $42 \cdot 2$ & 3.3 & $49 \cdot 3$ & 4.5 & $42 \cdot 7$ & 3.9 \\
\hline Noradrenaline (pg/ml) & $517 \cdot 1$ & 28.9 & $379 \cdot 5^{\star *}$ & $19 \cdot 9$ & $336 \cdot 8^{\star *}$ & $15 \cdot 3$ \\
\hline Dopamine $(\mathrm{pg} / \mathrm{ml})$ & 11.4 & 0.7 & 8.9 & 0.9 & $5 \cdot 8^{\star *} \dagger$ & 1.0 \\
\hline
\end{tabular}

FBG, fasting blood glucose; 5-HIAA, 5-hydroxyindoleacetic acid.

Mean values were significantly different from those of week $0:{ }^{*} P<0.05,{ }^{* \star} P<0.001$

Mean values were significantly different from those of week $4: \dagger P<0.05$.

‡For details of procedures, see Subjects and methods.

trial (Table 5). Although not statistically significant because of the presence of a single person with an extremely high value $(508 \mathrm{pg} / \mathrm{ml})$ at week 8 , serum MCP-1 exhibited a similar pattern. Lower ( $v$. baseline) sVCAM-1 and MCP-1 values at week 4 were observed in sixteen and seventeen of the twenty subjects, respectively. No reductions on SSE supplementation were seen in sICAM-1 and high-sensitivity C-reactive protein levels. A slight, but significant, increase of sICAM-1 was seen after the 4-week washout period.

\section{Discussion}

We previously reported that SSE and its major and unique phenolic constituents, serotonin hydroxycinnamic acid amides (serotonin derivatives), were protective against LDL oxidation and atherogenesis ${ }^{(9)}$. Although safflower seeds have been used in herbal medicines in some countries, to the best of our knowledge, human studies on its extracts or major components have not been reported. We questioned whether SSE has the potential to safely modulate oxidative stress and act as a cardioprotective in man as it did in experimental animals. To answer this question, we conducted

Table 3. Effects of safflower seed extract supplementation on brachial-ankle pulse wave velocity (baPWV) and blood pressure* (Mean values with their standard errors)

\begin{tabular}{|c|c|c|c|c|c|c|}
\hline & \multicolumn{2}{|c|}{ Week 0 (n 20) } & \multicolumn{2}{|c|}{ Week 4 (n 20) } & \multicolumn{2}{|c|}{$\begin{array}{l}\text { Week } 8 \text { (after } \\
\text { washout, } n 19 \text { ) }\end{array}$} \\
\hline & Mean & SEM & Mean & SEM & Mean & SEM \\
\hline baPWV $(\mathrm{cm} / \mathrm{s})$ & $1318 \cdot 6$ & 26.9 & $1298 \cdot 3$ & $22 \cdot 7$ & $1296 \cdot 7$ & $26 \cdot 8$ \\
\hline $\mathrm{SBP}(\mathrm{mmHg})$ & 125.9 & $3 \cdot 1$ & 123.5 & $3 \cdot 1$ & $123 \cdot 1$ & $2 \cdot 4$ \\
\hline $\mathrm{DBP}(\mathrm{mmHg})$ & $78 \cdot 0$ & $2 \cdot 0$ & $76 \cdot 0$ & 1.9 & 75.5 & 1.7 \\
\hline $\mathrm{PP}(\mathrm{mmHg})$ & $47 \cdot 9$ & $2 \cdot 1$ & 47.5 & $2 \cdot 0$ & $47 \cdot 6$ & 1.7 \\
\hline $\mathrm{MBP}(\mathrm{mmHg})$ & $95 \cdot 8$ & $2 \cdot 2$ & 93.9 & $2 \cdot 4$ & 93.6 & $2 \cdot 2$ \\
\hline
\end{tabular}

$\mathrm{DBP}$, diastolic blood pressure; MBP, mean blood pressure; $\mathrm{PP}$, pulse pressure;

SBP, systolic blood pressure.

${ }^{\star}$ For details of procedures, see Subjects and methods. a non-placebo-controlled human pilot study in which its effects on various oxidative stress markers and cardiovascular risk factors and also on some toxicological parameters were examined. In addition, we looked into the possible effect of SSE on haemodynamics, especially on PWV, one of the non-invasive measures of aortic stiffness, because SSE and serotonin derivatives both ameliorated aortic PWV in cholesterol-fed KHC rabbits ${ }^{(15)}$.

Consistent with the result of our 4-week safety evaluation in rats conducted prior to the present study (data not shown), SSE was well tolerated in every participant and no subjective symptoms or significant changes in blood biochemical parameters for hepatic and renal functions were observed at the completion of the 4-week intervention. These results indicate that at least up to $2.1 \mathrm{~g}$ intake of SSE daily (including $1.05 \mathrm{~g}$ $\gamma$-cyclodextrin as vehicle) for 4 weeks is safe. Blood lipids and glucose were not affected by the intervention (Table 2), while a significant reduction in total cholesterol was observed in apoE-deficient mice fed on SSE- or serotonin derivativesupplemented diets for 15 weeks ${ }^{(9)}$. Differences in species, intervention period and/or dosage might have contributed to this discrepancy.

In the present study, to avoid too much blood sample collection, we did not directly measure the plasma concentration of serotonin derivatives to confirm their biological availability in man. However, a characteristic change in whole-blood serotonin in association with SSE intake was observed in most participants (Table 2). We interpreted the present result as being indicative of in vivo exposure to absorbed serotonin derivatives for the following reasons: (1) serotonin derivatives in SSE have been shown to be absorbed into the circulation in mice ${ }^{(9)}$; (2) serotonin derivatives harbour a serotonin moiety in their own structures, and it is probable that some absorbed serotonin derivatives are hydrolysed at their amide bond to liberate serotonin; (3) only serotonin was elevated while other biogenic monoamines were not; (4) such elevation was reproducible in mice orally administered with synthetic serotonin derivatives for 2 weeks or longer (but not reproduced by green tea catechin administration), and the extent was 
Table 4. Effects of safflower seed extract supplementation on oxidative stress-related biomarkersł (Mean values with their standard errors)

\begin{tabular}{|c|c|c|c|c|c|c|}
\hline & \multicolumn{2}{|c|}{ Week 0 (n 20) } & \multicolumn{2}{|c|}{ Week 4 ( $n$ 20) } & \multicolumn{2}{|c|}{ Week 8 (after washout, $n$ 19) } \\
\hline & Mean & SEM & Mean & SEM & Mean & SEM \\
\hline $\operatorname{oxLDL}(\mathrm{U} / \mathrm{l})$ & 65.9 & 3.4 & $56 \cdot 9^{*}$ & $3 \cdot 8$ & $58 \cdot 9^{*}$ & 3.8 \\
\hline anti-MDA-LDL (AU) & $112 \cdot 0$ & 9.9 & $76 \cdot 5^{*}$ & 5.9 & $88.8^{*}$ & 8.8 \\
\hline \multicolumn{7}{|l|}{ TBARS } \\
\hline in serum $(\mu \mathrm{mol} / \mathrm{l})$ & 5.4 & 0.3 & $5 \cdot 3$ & 0.3 & $5 \cdot 3$ & 0.3 \\
\hline in LDL (nmol/mg protein) & 0.32 & 0.10 & 0.26 & 0.07 & 0.29 & 0.02 \\
\hline 8-Isoprostane $(\mathrm{ng} / \mathrm{mmol}) \S$ & $20 \cdot 6$ & $1 \cdot 1$ & $11 \cdot 6^{\star \star}$ & 0.8 & $14 \cdot 9^{\star \star} \dagger \dagger$ & $1 \cdot 3$ \\
\hline 8-OHdG $(\mu \mathrm{g} / \mathrm{mmol}) \S$ & 1.3 & 0.1 & $1 \cdot 2$ & 0.1 & $1 \cdot 1$ & 0.1 \\
\hline Protein carbonyls ( $\mathrm{nmol} / \mathrm{mg}$ protein) & $1 \cdot 10$ & 0.03 & $1 \cdot 15$ & 0.02 & 1.27 & 0.02 \\
\hline \multicolumn{7}{|l|}{ Serum antioxidative enzyme activity } \\
\hline Superoxide dismutase $(\mathrm{U} / \mathrm{ml})$ & 2.9 & 0.5 & $2 \cdot 8$ & 0.5 & 3.1 & 0.6 \\
\hline Paraoxonase $(\mathrm{U} / \mathrm{I})$ & 324.6 & 24.4 & 326.9 & $20 \cdot 1$ & $347 \cdot 2^{*} \dagger$ & 23.1 \\
\hline
\end{tabular}

anti-MDA-LDL, autoantibody titres to malondialdehyde-modified LDL; AU, arbitrary unit; oxLDL, oxidized LDL; 8-OHdG, 8-hydroxydeoxyguanosine; TBARS, thiobarbituric acid-reactive substances.

Mean values were significantly different from those of week $0:{ }^{*} P<0.05,{ }^{*} P<0.001$.

Mean values were significantly different from those of week 4 : $\dagger P<0.05, \dagger \dagger P<0.001$

$\ddagger$ For details of procedures, see Subjects and methods.

$\S$ Normalized by urinary creatinine.

proportional to the dose of serotonin derivative (unpublished results). If this speculation is correct, the elevation of whole-blood serotonin observed in the present study may be a reflection of the biological availability of serotonin derivatives. It should be noted that, although there was a significant response in whole-blood serotonin, its concentration did not exceed the upper limit of the reference range $(230 \mathrm{ng} / \mathrm{ml})$ for any of the participants. Therefore, it seems unlikely that slightly, but significantly elevated serotonin is responsible for every in vivo effect of absorbed serotonin derivatives. However, a more detailed pharmacokinetic study on the fate of ingested serotonin derivatives and their relevance to the serotonin level in whole blood and the in vivo effect of serotonin derivatives is required to confirm the assumptions given earlier. Although not human studies, two rat pharmacokinetic studies on CS and $N$-feruloylserotonin have been reported at recent scientific meetings ${ }^{(22,23)}$. In these studies, rapid absorption $\left(\mathrm{T}_{\max } \leq 15 \mathrm{~min}\right)$ and relatively high blood concentrations $\left(\mathrm{C}_{\max }=10-40 \mu \mathrm{M}\right)$ of these

Table 5. Effects of safflower seed extract supplementation on serum biomarkers of inflammation $\ddagger$

(Mean values with their standard errors)

\begin{tabular}{|c|c|c|c|c|c|c|}
\hline & \multicolumn{2}{|c|}{ Week 0 (n 20) } & \multicolumn{2}{|c|}{ Week 4 ( $n$ 20) } & \multicolumn{2}{|c|}{$\begin{array}{l}\text { Week } 8 \text { (after } \\
\text { washout, } n \text { 19) }\end{array}$} \\
\hline & Mean & SEM & Mean & SEM & Mean & SEM \\
\hline sVCAM-1 (ng/ml) & 578.9 & 33.5 & $519 \cdot 2^{*}$ & $27 \cdot 5$ & 552.4 & 27.5 \\
\hline sICAM-1 (ng/ml) & $216 \cdot 3$ & $32 \cdot 8$ & $217 \cdot 7$ & $32 \cdot 3$ & $229 \cdot 5^{*} \dagger$ & $35 \cdot 6$ \\
\hline MCP-1 (pg/ml) & $212 \cdot 0$ & 9.6 & $186 \cdot 6$ & $9 \cdot 0$ & 194.5 & $19 \cdot 4$ \\
\hline hsCRP (ng/ml) & $605 \cdot 2$ & 209.9 & $797 \cdot 2$ & $367 \cdot 3$ & $822 \cdot 2$ & $268 \cdot 1$ \\
\hline
\end{tabular}

hsCRP, high-sensitivity C-reactive protein; MCP-1, monocyte chemotactic protein-1; sICAM-1, soluble form of intercellular adhesion molecule-1; sVCAM-1, soluble form of vascular cell adhesion molecule-1.

Mean values were significantly different from those of week $0:{ }^{*} P<0.05$.

Mean values were significantly different from those of week $4: \dagger P<0.05$

$\ddagger$ For details of procedures, see Subjects and methods. compounds have been demonstrated in rats administered CS or $N$-feruloylserotonin orally at $100 \mathrm{mg} / \mathrm{kg}$. Because some indoles such as tryptophan and indoleamines can reach relatively high physiological concentrations along with dietary intake in man ${ }^{(4)}$, we speculated that CS and $\mathrm{N}$-feruloylserotonin may also have good biological availability in man.

There were no significant changes in baPWV, systolic BP and diastolic BP during the study period (Table 3); however, lower ( $v$. baseline) week $4 \mathrm{baPWV}$ values were seen in eleven of twenty subjects. In this 'baPWV-reduced group', mean baPWV at baseline and week 4 were 1362.3 (SEM $23 \cdot 1$ ) and $1296 \cdot 3$ (SEM 25.9) $\mathrm{cm} / \mathrm{s}$, respectively. This change was accompanied by a reduction of diastolic BP (from 81.2 (SEM 1.9) to 75.9 (SEM 1.4) $\mathrm{mmHg}, P<0.05$ ) and mean BP (from 98.5 (SEM 2.4) to 94.8 (SEM 2.4) $\mathrm{mmHg}, P<0.05$ ). Similarly, simultaneous significant reductions in both systolic BP and aortic PWV have been seen in cholesterol-fed KHC rabbits supplemented with $4 \%(w / w)$ SSE for 4 weeks (S Katsuda, unpublished results). Because of the nature of PWV, which reflects not only the structural properties but also the functional properties of arteries and is significantly influenced by $\mathrm{BP}^{(24)}$, the authors suspected that SSE affected vascular function by reducing $\mathrm{BP}$ which resulted in a reduction of baPWV in certain cases in this relatively short-term study. Further analysis revealed that baPWV decreased significantly $(P<0.05)$ in stratified groups with relatively high initial BP (diastolic BP $\geq 85 \mathrm{mmHg}, n$ 6), with relatively high initial baPWV $(\geq 1300 \mathrm{~cm} / \mathrm{s}, n 14)$, and with age of 35 years and older ( $n$ 10). This information may suggest that older subjects with elevated BP and/or arterial stiffness may receive more benefit from SSE supplementation. To confirm this hypothesis, a further study involving a large number of subjects who fall into such a category needs to be conducted in a placebocontrolled randomized fashion in parallel with a detailed study on the possible effects of SSE or serotonin derivatives on vascular tone.

It is noteworthy that multiple biomarkers for oxidative stress and inflammation were affected by SSE supplementation. 
Reductions of serum oxLDL, anti-MDA-LDL, urinary 8-isoprostane, serum sVCAM-1 and MCP-1 from baseline were observed in fifteen, sixteen, twenty, sixteen and seventeen of the twenty subjects, respectively, after 4-week SSE supplementation. The finding is in part consistent with our previous demonstration that autoantibody titres to oxLDL and lipid peroxide in plasma were significantly reduced in apoE-deficient mice fed on SSE or serotonin derivative-supplemented diets ${ }^{(9)}$. Circulating autoantibody titres have been shown to correlate with oxLDL content in atherosclerotic lesions of LDL-receptor-deficient mice ${ }^{(25)}$; however, as oxLDL in circulation is very difficult to detect in mice, the inhibition of oxLDL formation in vivo has remained to be confirmed as a possible mechanism of the reduction of autoantibody titres. The parallel reduction of serum oxLDL and autoantibody titres observed in the present study may support the proposed mechanism. 8-Isoprostane is one of the specific end products of the peroxidation of arachidonic acid and is considered a reliable marker of systemic oxidative stress ${ }^{(26)}$, and a sensitive and independent risk marker of $\mathrm{CHD}^{(10)}$. The significant reduction of 8 -isoprostane in conjunction with oxLDL and anti-MDA-LDL strongly suggested that SSE has antioxidant effects in man as well as in apoE-deficient mice. These are not only markers of oxidative stress but also have been shown to have various biological activities, and a causative effect on CVD has been proposed ${ }^{(26,27)}$. Thus, the lowering effect on oxLDL and 8-isoprostane may have a substantial role in the antiatherogenic action of SSE.

Migration of circulating monocytes into the subendothelial space is recognized as one of the crucial events in early atherogenesis. ICAM-1, VCAM-1 and MCP-1 are wellknown factors involved in this process ${ }^{(28)}$. Kawashima et al. ${ }^{(29)}$ reported that CS suppressed proinflammatory cytokine (TNF- $\alpha$, IL-1 $\alpha, \beta$, IL-6) production from lipopolysaccharide-stimulated human monocytes in vitro. They proposed the inhibitory effect of CS on NF-кB activation as an underlying mechanism for this. Because NF- $\kappa \mathrm{B}$ is activated by reactive oxygen species, the radical scavenging activity of CS may have contributed to the inhibitory effect on NF- $\mathrm{KB}$ activation. Expression of ICAM-1, VCAM-1 and MCP-1 are also regulated by the NF-кB system ${ }^{(30)}$. Therefore, it may not be surprising that some of these are decreased by the intake of SSE, which contains $\mathrm{CS}$ and $\mathrm{N}$-feruloylserotonin as its major phenolic constituents. The reason for the increase of sICAM-1 after the washout at week 8 is not clear at this time; however, it seems unlikely that it can be considered as a harmful effect of SSE because the increase was slight, not observed at the end of the intervention (week 4) and the serum level at week 8 was apparently within the physiological range in healthy Japanese subjects ${ }^{(31)}$. In the present study, we did not measure any adipocytokines since the subjects were non-obese. However, as adipocytokines have a significant role in proinflammatory reactions and atherogenesis, it might be interesting to look into the effects of SSE on them and to analyse if there is any relevance to the favourable effects of SSE noted earlier.

To estimate which populations are more susceptible to the favourable effects of SSE, we examined whether the intervention had an effect on cardiovascular risk. Statistically significant negative correlations were found between baseline values and the changes from baseline at week 4 in the following parameters; baPWV (Fig. 1 (A)), 8-isoprostane (Fig. 1 (B)), anti-MDA-LDL (Fig. 1 (C)) and sVCAM-1 (Fig. 1 (D)). MCP-1 was marginally non-significant $(r-0.415, P=0.0686)$. If the change of the autoantibody titres is secondary to that of oxLDL content in a body, it is somewhat conflicting that a considerably strong negative correlation between the initial risk and the intervention effect was seen in autoantibody titres, whereas no significant relationship was found in circulating oxLDL $(r-0.334, P=0 \cdot 15)$. This may be explained by the possibility that the circulating oxLDL measured in the present study poorly reflected the
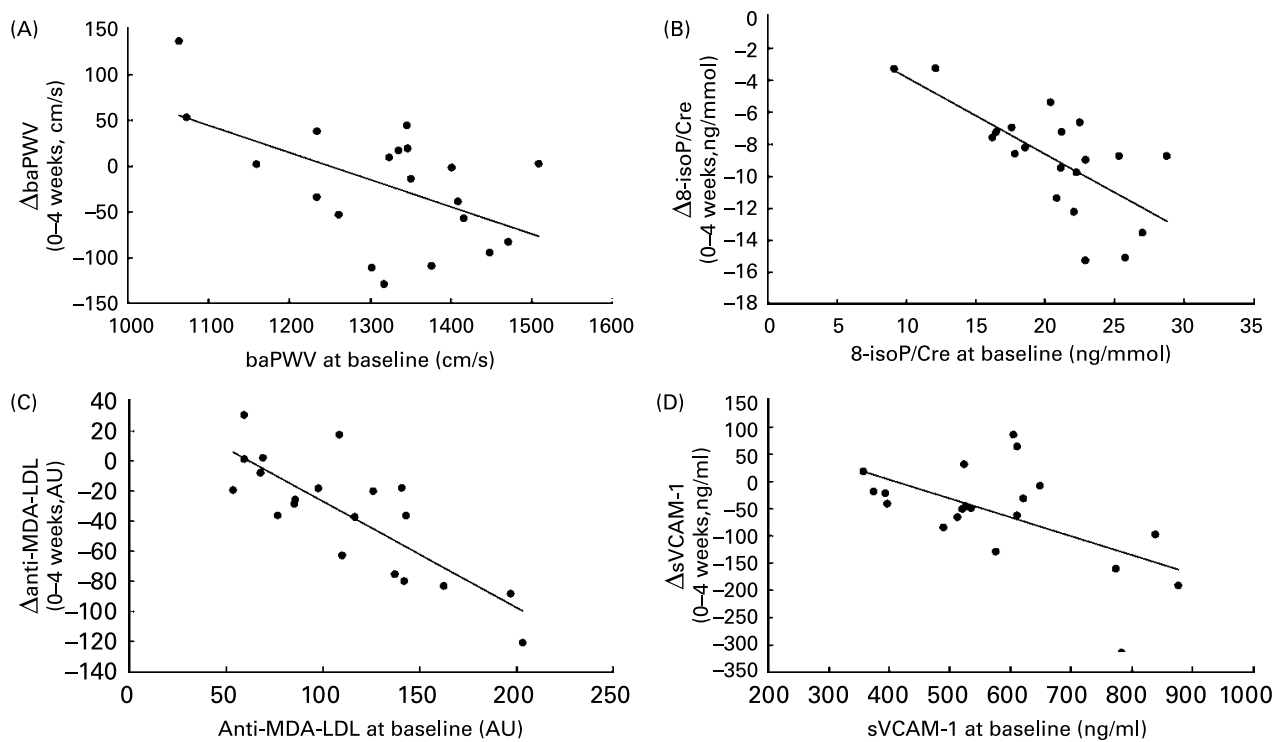

Fig. 1. Relationships between initial risk values (week 0 ) and safflower seed extract intervention effects (changes from baseline at week 4 ) on corresponding risk factors. (A), Brachial-ankle pulse wave velocity (baPWV; $r-0.541, P=0.0137)$; (B) 8-isoprostane/creatinine (8-iso/Cre; $r-0.693, P=0.000697) ;(C)$ autoantibody titres to malondialdehyde-modified LDL (anti-MDA-LDL; $r-0.807, P=0.0000168$ ); (D) soluble form of vascular cell adhesion molecule-1 (sVCAM-1; $r-0.574$, $P=0.0082$ ). 
total amount of oxLDL existing in a body relative to autoantibody titres in serum, or that SSE had a direct effect on the immune system, which is responsible for antibody production. The analysis given earlier may suggest that SSE may be more beneficial to individuals with elevated cardiovascular risk(s) especially related to oxidative stress and inflammation, and also with stiffened arteries.

In conclusion, the present pilot study suggested that SSE is safe and has the potential to protect human subjects from oxidative insult and proinflammatory reactions. Although there were no significant changes in BP or baPWV when the analysis was conducted on the entire cohort, a favourable effect on baPWV could be seen in certain individuals with relatively high $\mathrm{BP}$, or with relatively high baPWV, or with an age of 35 years and older. Moreover, it was suggested that individuals with elevated cardiovascular risk factors such as 8-isoprostane, anti-MDA-LDL, sVCAM-1 in serum may receive more benefit from SSE supplementation. To confirm whether SSE is protective on arterial stiffening and actually reduces cardiovascular risk, a further longer-term study involving a larger number of subjects with high cardiovascular risk needs to be conducted in a placebo-controlled randomized manner. The information given concerning populations benefiting from SSE may facilitate the appropriate design of future clinical trials.

\section{Acknowledgements}

The authors are employees of Ajinomoto Co. Inc. and the work described in this article has been funded by the company. The authors declare that they have no other conflict of interest.

N. K. and E. S. designed the human study. T. S. and K. I. provided SSE for this study. N. K., K. S., Y. F., H. A. and $\mathrm{K}$. K. conducted the human study (Y. F. is the responsible physician in this study). K. S. and N. K. did the data analysis. N. K. drafted the paper. M. T. supervised the entire work. The authors are grateful to Satoko Tsuji and to Tomoka Yamano for assistance with the human study and for providing help with thiobarbituric acid-reactive substances measurement (Tomoka Yamano). We also thank Hiroshi Kuhara for providing helpful technical advice on large-scale preparation of SSE.

\section{References}

1. O’Reilly JD, Mallet AI, McAnlis GT, Young IS, Halliwell B, Sanders TA \& Wiseman H (2001) Consumption of flavonoids in onions and black tea: lack of effect on F2-isoprostanes and autoantibodies to oxidized LDL in healthy humans. Am J Clin Nutr 73, 1040-1044.

2. Mathur S, Devaraj S, Grundy SM \& Jialal I (2002) Cocoa products decrease low density lipoprotein oxidative susceptibility but do not affect biomarkers of inflammation in humans. J Nutr 132, 3663-3667.

3. Halliwell B, Rafter J \& Jenner A (2005) Health promotion by flavonoids, tocopherols, tocotrienols, and other phenols: direct or indirect effects? Antioxidant or not? Am J Clin Nutr 81, 268S-276S.

4. Herraiz T \& Galisteo J (2004) Endogenous and dietary indoles: a class of antioxidants and radical scavengers in the ABTS assay. Free Radic Res 38, 323-331.
5. Fukutomi J, Fukuda A, Fukuda S, Hara M, Terada A \& Yoshida M (2006) Scavenging activity of indole compounds against cisplatininduced reactive oxygen species. Life Sci 80, 254-257.

6. Vincenzi FF \& Hinds TR (1999) Stobadine: bellwether of a broader view of drug actions. Life Sci 65, 1857-1864.

7. Hotta Y, Nagatsu A, Liu W, et al. (2002) Protective effects of antioxidative serotonin derivatives isolated from safflower against postischemic myocardial dysfunction. Mol Cell Biochem 238, 151-162.

8. Kim EO, Oh JH, Lee SK, Lee JY \& Choi SW (2007) Antioxidant properties and quantification of phenolic compounds from safflower (Carthamus tinctorius L.) seeds. Food Sci Biotechnol 16, 71-77.

9. Koyama N, Kuribayashi K, Seki T, Kobayashi K, Furuhata Y, Suzuki K, Arisaka H, Nakano T, Amino Y \& Ishii K (2006) Serotonin derivatives, major safflower (Carthamus tinctorius L.) seed antioxidants, inhibit low-density lipoprotein (LDL) oxidation and atherosclerosis in apolipoprotein E-deficient mice. J Agric Food Chem 54, 4970-4976.

10. Schwedhelm E, Bartling A, Lenzen H, Tsikas D, Maas R, Brummer J, Gutzki FM, Berger J, Frolich JC \& Boger RH (2004) Urinary 8-iso-prostaglandin F2alpha as a risk marker in patients with coronary heart disease: a matched case-control study. Circulation 109, 843-848.

11. Holvoet P, Vanhaecke J, Janssens S, Van de Werf F \& Collen D (1998) Oxidized LDL and malondialdehyde-modified LDL in patients with acute coronary syndromes and stable coronary artery disease. Circulation 98, 1487-1494.

12. Bui MN, Sack MN, Moutsatsos G, Lu DY, Katz P, McCown R, Breall JA \& Rackley CE (1996) Autoantibody titers to oxidized low-density lipoprotein in patients with coronary atherosclerosis. Am Heart J 131, 663-667.

13. London GM \& Cohn JN (2002) Prognostic application of arterial stiffness: task forces. Am J Hypertens 15, 754-758.

14. Yambe M, Tomiyama H, Hirayama Y, Gulniza Z, Takata Y, Koji Y, Motobe K \& Yamashina A (2004) Arterial stiffening as a possible risk factor for both atherosclerosis and diastolic heart failure. Hypertens Res 27, 625-631.

15. Katsuda S, Koyama N, Suzuki K, Takahashi M, Takazawa K, Miyake M \& Hazama A (2007) Safflower seed serotonin derivatives improve aortic pulse wave velocity and augmentation index in Kurosawa and Kusanagi-hypercholesterolemic rabbits. $J$ Clin Biochem Nutr 41, Suppl., 118.

16. Singleton V, Orthofer R \& Lamuela-Raventós RM (1999) Analysis of total polyphenols and other oxidation substrates and antioxidants by means of Folin-Ciocalteu reagent. Methods Enzymol 299, 152-178.

17. Yamashina A, Tomiyama H, Takeda K, Tsuda H, Arai T, Hirose K, Koji Y, Hori S \& Yamamoto Y (2002) Validity, reproducibility, and clinical significance of noninvasive brachial-ankle pulse wave velocity measurement. Hypertens Res 25, 359-364.

18. Levine RL, Garland D, Oliver CN, Amici A, Climent I, Lenz AG, Ahn BW, Shaltiel S \& Stadtman ER (1990) Determination of carbonyl content in oxidatively modified proteins. Methods Enzymol 186, 464-478.

19. Ito $\mathrm{Y}$, Nakachi K, Imai K, Hashimoto S, Watanabe $\mathrm{Y}$, Inaba $\mathrm{Y}$, Tamakoshi A \& Yoshimura T (2005) Stability of frozen serum levels of insulin-like growth factor-I, insulin-like growth factor-II, insulin-like growth factor binding protein-3, transforming growth factor beta, soluble Fas, and superoxide dismutase activity for the JACC study. J Epidemiol 15, Suppl. 1, S67-S73.

20. Ohkawa H, Ohishi N \& Yagi K (1979) Assay for lipid peroxides in animal tissues by thiobarbituric acid reaction. Anal Biochem 95, 351-358.

21. Chung BH, Segrest JP, Ray MJ, Brunzell JD, Hokanson JE, Krauss RM, Beaudrie K \& Cone JT (1986) Single vertical 
spin density gradient ultracentrifugation. Methods Enzymol $\mathbf{1 2 8}$ $181-209$.

22. Tanno K, Ichiyanagi T, Ikeshiro Y \& Hatano Y (2007) Pharmacokinetic behavior of serotonin derivatives, major antioxidants in safflower seed. J Clin Biochem Nutr 41, Suppl., 94.

23. Shirai Y, Ueno S, Ikeuchi K \& Mihara R (2007) Pharmacokinetic study of $N$-feruloylserotonin and $N$-( $p$-coumaroyl)serotonin in rats. In $3 r$ International Conference on Polyphenols and Health (Program \& Abstracts ICPH2007), p. 193. Japan: ICPH 2007 Organizing Committee.

24. Tomiyama H, Yamashina A, Arai T, Hirose K, Koji Y, Chikamori T, Hori S, Yamamoto Y, Doba N \& Hinohara S (2003) Influences of age and gender on results of noninvasive brachial-ankle pulse wave velocity measurement - a survey of 12517 subjects. Atherosclerosis 166, 303-309.

25. Tsimikas S, Palinski W \& Witztum JL (2001) Circulating autoantibodies to oxidized LDL correlate with arterial accumulation and depletion of oxidized LDL in LDL receptor-deficient mice. Arterioscler Thromb Vasc Biol 21, 95-100.

26. Cracowski JL, Durand T \& Bessard G (2002) Isoprostanes as a biomarker of lipid peroxidation in humans: physiology, pharmacology and clinical implications. Trends Pharmacol Sci 23, $360-366$.
27. Palinski W \& Witztum JL (2000) Immune responses to oxidative neoepitopes on LDL and phospholipids modulate the development of atherosclerosis. J Intern Med 247, 371-380.

28. Piga R, Naito Y, Kokura S, Handa O \& Yoshikawa T (2007) Short-term high glucose exposure induces monocyte-endothelial cells adhesion and transmigration by increasing VCAM-1 and MCP-1 expression in human aortic endothelial cells. Atherosclerosis 193, 328-334.

29. Kawashima S, Hayashi M, Takii T, Kimura H, Zhang HL, Nagatsu A, Sakakibara J, Murata K, Oomoto Y \& Onozaki K (1998) Serotonin derivative, $N$-( $p$-coumaroyl) serotonin, inhibits the production of TNF-alpha, IL-1alpha, IL-1beta, and IL-6 by endotoxin-stimulated human blood monocytes. J Interferon Cytokine Res 18, 423-428.

30. Thurberg BL \& Collins T (1998) The nuclear factor-kappa B/ inhibitor of kappa B autoregulatory system and atherosclerosis. Curr Opin Lipidol 9, 387-396.

31. Yasuda H, Suzuki T, Zayasu K, Ishizuka S, Kubo H, Sasaki T, Nishimura H, Sekizawa K \& Yamaya M (2005) Inflammatory and bronchospastic factors in asthma exacerbations caused by upper respiratory tract infections. Tohoku J Exp Med 207, $109-118$ 